Bull. Korean Math. Soc. 50 (2013), No. 5, pp. 1433-1439

http://dx.doi.org/10.4134/BKMS.2013.50.5.1433

\title{
MORPHIC PROPERTY OF A QUOTIENT RING OVER POLYNOMIAL RING
}

\author{
Kai Long, Qichuan Wang, And Lianggui Feng
}

\begin{abstract}
A ring $R$ is called left morphic if $R / R a \cong l(a)$ for every $a \in R$. Equivalently, for every $a \in R$ there exists $b \in R$ such that $R a=l(b)$ and $l(a)=R b$. A ring $R$ is called left quasi-morphic if there exist $b$ and $c$ in $R$ such that $R a=l(b)$ and $l(a)=R c$ for every $a \in R$. A result of T.-K. Lee and Y. Zhou says that $R$ is unit regular if and only if $R[x] /\left(x^{2}\right) \cong R \propto R$ is morphic. Motivated by this result, we investigate the morphic property of the ring $S_{n} \stackrel{\text { def }}{=} R\left[x_{1}, x_{2}, \ldots, x_{n}\right] /\left(\left\{x_{i} x_{j}\right\}\right)$, where $i, j \in\{1,2, \ldots, n\}$. The morphic elements of $S_{n}$ are completely determined when $R$ is strongly regular.
\end{abstract}

\section{Introduction}

Morphic rings were first introduced by W. K. Nicholson and E. Sánchez Campos in [6]. A ring $R$ is called left morphic if $R / R a \cong l(a)$ for every $a \in R$. Equivalently, for every $a \in R$, there exists $b \in R$ such that $R a=l(b)$ and $l(a)=R b[6$, Lemma 1]. Right morphic rings are defined analogously. A left and right morphic ring is simply called a morphic ring. If there exist $b, c \in R$ such that $R a=l(b)$ and $l(a)=R c$, the element $a$ is called left quasi-morphic [1]. Morphic and quasi-morphic rings were discussed in great detail in [1], [6] and [7]. The morphic property of the trivial extension $R \propto M$ of a ring $R$ with a bimodule $M$ over $R$ is discussed in [2]. In particular, $R$ is unit regular if and only if $R[x] /\left(x^{2}\right)$ is morphic [5].

Motivated by these results, we investigate the morphic property of the ring $S_{n} \stackrel{\text { def }}{=} R\left[x_{1}, x_{2}, \ldots, x_{n}\right] /\left(\left\{x_{i} x_{j}\right\}\right)$, where $i, j \in\{1,2, \ldots, n\}$. By converting to the case of the elements of the type $\alpha=e+f x_{1}+a_{2} x_{2}+\cdots+a_{n} x_{n}$, where $e, f$ are idempotents in $R$, and $a_{i} \in(1-e) R(1-e), i \geq 2$, We completely determine the morphic elements of $S_{n}$ [Theorem 6]. Further, from the proof of this theorem, we know that the result is also right to left quasi-morphic

Received May 31, 2011.

2010 Mathematics Subject Classification. 16E50, 13F20, 16 U99.

Key words and phrases. morphic property, polynomial ring, strongly regular.

This study was supported by the National Natural Science Foundation of China(10926183) and the Foundation of National University of Defense Technology(JC08-2-03). 
elements in $S_{n}$ (Remark 1$)$. The case of $n=2$ has a very close relationship with trivial extension (Remark 2).

All rings here are associative with identity. The set of units of a ring $R$ is denoted by $U(R)$. We simply write $l(a)$ as its left annihilators, and $r(a)$ as its right annihilators. The $n \times n$ matrix ring over $R$ is denoted by $M_{n}(R)$. We write $\mathbb{Z}$ for the ring of integers and $\mathbb{Z}_{n}$ for integers module $n$, respectively. Regular rings here mean von Neumann regular rings.

\section{The unit elements in $R\left[x_{1}, x_{2}, \ldots, x_{n}\right] /\left(\left\{x_{i} x_{j}\right\}\right)$}

Denote $S_{n}=R\left[x_{1}, x_{2}, \ldots, x_{n}\right] /\left(\left\{x_{i} x_{j}\right\}\right), n \geq 2$, and $\alpha=a_{0}+\sum_{i=1}^{n} a_{i} x_{i}$ in $S_{n}$, where $a_{i} \in R, x_{i} x_{j}=0$, for all $i, j \in\{1,2, \ldots, n\}$ and $x_{i}$ commute with $R$. Throughout this article, we adopt this notation, and $n \geq 2$ is indispensable.

Lemma 1. Let $S_{n}$ and $R$ be as above, denote $U\left(S_{n}\right)$ as the set of units of ring $S_{n}$. Then $U\left(S_{n}\right)=\left\{u+\sum_{i=1}^{n} r_{i} x_{i} \mid u \in U(R), r_{i} \in R\right\}$.

Proof. First of all, the identity of $S_{n}$ is the same with $R$, we denote $1_{S_{n}}=1_{R}=$ 1. Assume $\alpha=a_{0}+\sum_{i=1}^{n} a_{i} x_{i}$ in $S_{n}$ is a unit, then there exists an element $\beta=a_{0}{ }^{\prime}+\sum_{i=1}^{n} a_{i}{ }^{\prime} x_{i}$ such that

$$
\begin{aligned}
\alpha \beta & =\left(a_{0}+\sum_{i=1}^{n} a_{i} x_{i}\right)\left(a_{0}{ }^{\prime}+\sum_{i=1}^{n} a_{i}{ }^{\prime} x_{i}\right) \\
& =a_{0} a_{0}{ }^{\prime}+\sum_{i=1}^{n}\left(a_{0} a_{i}{ }^{\prime}+a_{0}{ }^{\prime} a_{i}\right) x_{i}=1 .
\end{aligned}
$$

Hence we have $a_{0} a_{0}{ }^{\prime}=1$. Similarly, we can get $a_{0}{ }^{\prime} a_{0}=1$ by considering $\beta \alpha=1$. Thus $U\left(S_{n}\right) \subset\left\{u+\sum_{i=1}^{n} r_{i} x_{i} \mid u \in U(R), r_{i} \in R\right\}$.

Conversely, assume $u v=v u=1$, then

$$
\left(u+\sum_{i=1}^{n} r_{i} x_{i}\right)\left(v-\sum_{i=1}^{n} v r_{i} v x_{i}\right)=\left(v-\sum_{i=1}^{n} v r_{i} v x_{i}\right)\left(u+\sum_{i=1}^{n} r_{i} x_{i}\right)=1 .
$$

Thus $U\left(S_{n}\right) \supset\left\{u+\sum_{i=1}^{n} r_{i} x_{i} \mid u \in U(R), r_{i} \in R\right\}$. Hence the proof is completed.

If $R$ is unit regular, we have the following result, which can help us to convert $\alpha \in S_{n}$ into a simpler form.

Claim 1. Let $R$ be a unit regular ring. Then for any $\alpha=a_{0}+\sum_{i=1}^{n} a_{i} x_{i}$ in $S_{n}$, we have $\alpha=u_{s_{n}}\left(e+f x_{1}+a_{2} x_{2}+\cdots+a_{n} x_{n}\right) v_{s_{n}}$, where $u_{s_{n}}, v_{s_{n}} \in U\left(S_{n}\right)$, $e, f$ are idempotents in $R$, and $f, a_{i} \in(1-e) R(1-e), i \geq 2$.

Proof. Since $R$ is unit regular, every element of $R$ is the product of a unit and an idempotent. By multiplying $\alpha$ with a suitable unit of $R$ we can assume $\alpha=e_{0}+a_{1} x_{1}+\cdots+a_{n} x_{n}$. Then we have

$$
\prod_{i=0}^{n-1}\left(1-\left(1-e_{0}\right) a_{n-i} x_{n-i}\right) \cdot \alpha \cdot \prod_{i=1}^{n}\left(1-a_{i} x_{i}\right)
$$




$$
=e_{0}+\left(1-e_{0}\right) a_{1}\left(1-e_{0}\right) x_{1}+\cdots+\left(1-e_{0}\right) a_{n}\left(1-e_{0}\right) x_{n} .
$$

We also have $\left(1-e_{0}\right) a_{1}\left(1-e_{0}\right)=u_{0} f, u_{0} \in U\left(\left(1-e_{0}\right) R\left(1-e_{0}\right)\right), f \in(1-$ $\left.e_{0}\right) R\left(1-e_{0}\right)$ is idempotent, since $\left(1-e_{0}\right) R\left(1-e_{0}\right)$ is unit regular by [3]. Further there exists an element $v_{0} \in U\left(\left(1-e_{0}\right) R\left(1-e_{0}\right)\right)$, such that $u_{0} v_{0}=1-e_{0}$. Hence $\left(e_{0}+u_{0}\right)\left(e_{0}+v_{0}\right)=e_{0}+u_{0} v_{0}=1$. That is to say $e_{0}+v_{0} \in U(R)$, then

$$
\left(e_{0}+v_{0}\right) \alpha=e_{0}+f x_{1}+a_{2}{ }^{\prime} x_{2}+\cdots+a_{n}{ }^{\prime} x_{n} .
$$

All the factors are units of $S_{n}$ by Lemma 1, thus the claim is proved.

Claim 2. Let $\alpha^{\prime}$ be an element of $S_{n}$ satisfying $\alpha^{\prime}=e+f x_{1}+a_{2} x_{2}+\cdots+a_{n} x_{n}$, where $e, f$ are idempotents in $R$, and $f, a_{i} \in(1-e) R(1-e), i \geq 2$. Then

$$
l\left(\alpha^{\prime}\right)=l(e) \cap l(f) \cap l\left(a_{2}\right) \cap \cdots \cap l\left(a_{n}\right)+l(e) x_{1}+l(e) x_{2}+\cdots+l(e) x_{n} .
$$

Proof. Assume $r_{0}+\sum_{i=1}^{n} r_{i} x_{i} \in l\left(\alpha^{\prime}\right)$, then

$$
\begin{aligned}
\left(r_{0}+\sum_{i=1}^{n} r_{i} x_{i}\right)\left(e+f x_{1}+\sum_{i=2}^{n} a_{i} x_{i}\right) & =r_{0} e+\left(r_{0} f+r_{1} e\right) x_{1}+\sum_{i=2}^{n}\left(r_{0} a_{i}+r_{i} e\right) x_{i} \\
& =0 .
\end{aligned}
$$

So we have

$$
r_{0} e=0, r_{0} f+r_{1} e=0, r_{0} a_{i}+r_{i} e=0, i \geq 2 .
$$

Noticing that $e f=f e=a_{i} e=e a_{i}=0, i \geq 2$ and $e^{2}=e$, by multiplying $e$ on the respective two sides of equations $(*)$, we get

$$
r_{0} e=r_{0} f=r_{0} a_{i}=0, r_{1} e=0, r_{i} e=0, i \geq 2 .
$$

Hence $l\left(\alpha^{\prime}\right) \subset l(e) \cap l(f) \cap l\left(a_{2}\right) \cap \cdots \cap l\left(a_{n}\right)+l(e) x_{1}+l(e) x_{2}+\cdots+l(e) x_{n}$, and it is a routine way to verify $l\left(\alpha^{\prime}\right) \supset l(e) \cap l(f) \cap l\left(a_{2}\right) \cap \cdots \cap l\left(a_{n}\right)+l(e) x_{1}+$ $l(e) x_{2}+\cdots+l(e) x_{n}$. Thus the result is established.

\section{The morphic elements in $S_{n}$}

The following lemma comes from the paper [1] of V. Camillo, W. K. Nicholson and Z. Wang.

Lemma 2 ([1]). Let $R$ be a left quasi-morphic ring. Then the intersection of finite principal left ideals of $R$ is again principal.

A ring is called strongly regular if $a \in a^{2} R$ for every $a \in R$ [4]. Strongly regular rings are unit regular, hence are morphic and quasi-morphic $[1,6]$. It is well known that $R$ is strongly regular if and only if $R$ is regular and every idempotent in $R$ is center.

Lemma 3. Let $R$ be a strongly regular ring. Then for any $a, b \in R, R a b \subset R a$, particularly if $b \in U, R a b=R a$.

Proof. Since $R$ is strongly regular, for any $a \in R$, there exist $u \in U(R)$ and an idempotent element $e$ such that $a=u e$ and $R a b=R u e b=R e b=R b e \subset R e=$ $R a$. Of course, if $b \in U$, then " $\subset$ " can be replaced by "=". 
Lemma 4. Let $R$ be a strongly regular ring. Then for any $\alpha \in S_{n}$, we have $l(\alpha)=l\left(e+f x_{1}+a_{2} x_{2}+\cdots+a_{n} x_{n}\right)$, where $e, f$ are idempotents in $R$, and $f, a_{i} \in(1-e) R(1-e), i \geq 2$.

Proof. By Claim 1, we have $\alpha=u_{s_{n}}\left(e+f x_{1}+a_{2} x_{2}+\cdots+a_{n} x_{n}\right) v_{s_{n}}$, where $u_{s_{n}}, v_{s_{n}} \in U\left(S_{n}\right)$, and $e, f$ are idempotents in $R$, and $f, a_{i} \in(1-e) R(1-e), i \geq$ 2. Since $u_{s_{n}}^{-1} \in U\left(S_{n}\right)$, by Lemma 1 , we can assume $u_{s_{n}}^{-1}=u+r_{1} x_{1}+\cdots+r_{n} x_{n}$, where $u \in U(R), r_{i} \in R, i=1,2, \ldots, n$. Then

$$
\begin{aligned}
l(\alpha)= & l\left(e+f x_{1}+a_{2} x_{2}+\cdots+a_{n} x_{n}\right) u_{s_{n}}^{-1} \\
= & \left\{\left(t_{0}+t_{1} x_{1}+\cdots+t_{n} x_{n}\right)\left(u+r_{1} x_{1}+\cdots+r_{n} x_{n}\right) \mid\right. \\
& \left.t_{0} \in l(e) \cap l(f) \cap l\left(a_{2}\right) \cap \cdots \cap l\left(a_{n}\right), t_{i} \in l(e), i \geq 1\right\} \\
= & t_{0} u+\left(t_{1} u+t_{0} r_{1}\right) x_{1}+\cdots+\left(t_{i} u+t_{0} r_{i}\right) x_{i}+\cdots \\
& +\left(t_{n} u+t_{0} r_{n}\right) x_{n} \mid t_{0} \in l(e) \cap l(f) \cap l\left(a_{2}\right) \cap \cdots \cap l\left(a_{n}\right), \\
& \left.t_{i} \in l(e), i \geq 1\right\} .
\end{aligned}
$$

By Lemma 2 and Lemma 3, we know that $t_{0} u, t_{0} r_{i} \in l(e) \cap l(f) \cap l\left(a_{2}\right) \cap$ $\cdots \cap l\left(a_{n}\right), t_{i} u \in l(e)$. Noticing that $l(e) \cap l(f) \cap l\left(a_{2}\right) \cap \cdots \cap l\left(a_{n}\right) \subset l(e)$, hence $t_{i} u+t_{0} r_{i}$ is in $l(e)$. Thus

$$
\begin{aligned}
l(\alpha) & =l\left(e+f x_{1}+a_{2} x_{2}+\cdots+a_{n} x_{n}\right) u_{s_{n}}^{-1} \\
& \subset l\left(e+f x_{1}+a_{2} x_{2}+\cdots+a_{n} x_{n}\right) .
\end{aligned}
$$

In fact, by the proof, we know that the equation $(* *)$ is also right for an arbitrary $u_{s_{n}}^{-1} \in U\left(S_{n}\right)$. Then

$$
\begin{aligned}
l\left(e+f x_{1}+a_{2} x_{2}+\cdots+a_{n} x_{n}\right) & =l\left(e+f x_{1}+a_{2} x_{2}+\cdots+a_{n} x_{n}\right) u_{s} u_{s}^{-1} \\
& \subset l\left(e+f x_{1}+a_{2} x_{2}+\cdots+a_{n} x_{n}\right) u_{s}^{-1} \\
& =l(\alpha) .
\end{aligned}
$$

Combing these together, we get $l(\alpha)=l\left(e+f x_{1}+a_{2} x_{2}+\cdots+a_{n} x_{n}\right)$, where $e, f$ are idempotents in $R$, and $f, a_{i} \in(1-e) R(1-e), i \geq 2$.

Above lemma tells us that if $\alpha=e+f x_{1}+a_{2} x_{2}+\cdots+a_{n} x_{n}$ is left morphic, and there is an element $\beta$ such that $S_{n} \alpha=l(\beta), l(\alpha)=S_{n} \beta$, then we can further assume that $\beta$ has the form of $e^{\prime}+f^{\prime} x_{1}+a_{2}{ }^{\prime} x_{2}+\cdots+a_{n}{ }^{\prime} x_{n}$, where $e^{\prime}, f^{\prime}$ are idempotents in $R$, and $f^{\prime}, a_{i}{ }^{\prime} \in\left(1-e^{\prime}\right) R\left(1-e^{\prime}\right), i \geq 2$.

Lemma 5. Let $R$ be a strongly regular ring, $\alpha=e+f x_{1}+a_{2} x_{2}+\cdots+a_{n} x_{n}$ in $S_{n}$, where $e, f$ are idempotents in $R$, and $f, a_{i} \in(1-e) R(1-e), i \geq 2$. Then $\alpha$ is left quasi-morphic $\Rightarrow R f=R a_{2}=\cdots=R a_{n}$.

Proof. If $e=1$, then $f, a_{i} \in(1-e) R(1-e)=0$, and $R f=R a_{2}=\cdots=R a_{n}=$ 0 . So we assume $\alpha$ is quasi-morphic and $e \neq 1$. By Lemma 4 and Claim 2, we have

$$
S_{n} \alpha=\left\{t_{0} e+\left(t_{1} e+t_{0} f\right) x_{1}+\cdots+\left(t_{i} e+t_{0} a_{i}\right) x_{i}+\cdots\right.
$$




$$
\begin{aligned}
& \left.+\left(t_{n} e+t_{0} a_{n}\right) x_{n} \mid t_{i} \in R\right\} \\
= & l(\beta) \\
= & l\left(e^{\prime}\right) \cap l\left(f^{\prime}\right) \cap l\left(a_{2}{ }^{\prime}\right) \cap \cdots \cap l\left(a_{n}{ }^{\prime}\right)+l\left(e^{\prime}\right) x_{1} \\
& +l\left(e^{\prime}\right) x_{2}+\cdots+l\left(e^{\prime}\right) x_{n} .
\end{aligned}
$$

Then there must be $R e+R f=R e+R a_{i}=l\left(e^{\prime}\right)$. Multiplying both sides by $1-e$, we get $R f=R a_{i}, i=2,3, \ldots, n$.

Theorem 6. Let $R$ be a strongly regular ring, denote by $T$ the set of morphic elements of ring $S_{n}$. Then $T=\left\{u_{s_{n}} e \mid u_{s_{n}} \in U\left(S_{n}\right), e^{2}=e \in R\right\}$.

Proof. Since multiplying units does not change the morphic property of an element [6], by Claim 1, we can assume $\alpha=e+f x_{1}+a_{2} x_{2}+\cdots+a_{n} x_{n}$ in $S_{n}$, where $e, f$ are idempotents in $R$, and $f, a_{i} \in(1-e) R(1-e), i \geq 2$.

By the proof of Lemma 5 , we get $R e=l\left(e^{\prime}\right) \cap l\left(f^{\prime}\right) \cap l\left(a_{2}{ }^{\prime}\right) \cap \cdots \cap l\left(a_{n}{ }^{\prime}\right)$, $R e+R f=R e+R a_{i}=l\left(e^{\prime}\right), i=2,3, \ldots, n$. Thus $l(\beta)=R e+\sum_{i=1}^{n}(R e+R f) x_{i}$. Considering the element of the type $\alpha_{0}=f x_{1}+e x_{2}$, since

$$
\begin{aligned}
\alpha_{0} & \in R e+(R e+R f) x_{1}+\cdots+(R e+R f) x_{n} \\
& =l(\beta)=S_{n} \alpha \\
& =\left\{t_{0} e+\left(t_{1} e+t_{0} f\right) x_{1}+\cdots+\left(t_{i} e+t_{0} a_{i}\right) x_{i}+\cdots+\left(t_{n} e+t_{0} a_{n}\right) x_{n} \mid t_{i} \in R\right\}
\end{aligned}
$$

so we have $f=t_{1} e+t_{0} f, e=t_{2} e+t_{0} a_{2}$.

Multiplying both sides by $e$, we get $t_{1} e=0, e=t_{2} e$, and $f=t_{0} f, t_{0} a_{2}=0$. By Lemma 5 and noticing that $R$ is strongly regular, we have $a_{2}=r f=f r$, then $R(1-f) a_{2}=R(1-f) f r=0$. That is to say $l(f) \subset l\left(a_{2}\right)$. Assume $1-t_{0}=r_{0}, r_{0} \in l(f)$, then $t_{0} a_{2}=\left(1-r_{0}\right) a_{2}=a_{2}=0$. Thus $R f=R a_{2}=$ $\cdots=R a_{n}=0$, so $f=a_{2}=\cdots=a_{n}=0$.

Hence $T \subset\left\{u_{s_{n}} e \mid u_{s_{n}} \in U\left(S_{n}\right), e^{2}=e \in R\right\}$. Since $T \supset\left\{u_{s_{n}} e \mid u_{s_{n}} \in\right.$ $\left.U\left(S_{n}\right), e^{2}=e \in R\right\}$ is trivial, we complete the proof.

Remark 1. In fact, our proof just uses the property that $R$ is strongly regular and $S_{n} \alpha=l(\beta)$, hence the theorem is also right for left quasi-morphic elements in $S_{n}$.

Remark 2 . The case of $n=2$ has a very close relationship with trivial extension, since

$$
R \propto(R \propto R) \cong\left\{\left(\begin{array}{llll}
a & 0 & b & c \\
0 & a & 0 & b \\
0 & 0 & a & 0 \\
0 & 0 & 0 & a
\end{array}\right) \mid a, b, c \in R\right\} \cong R[x, y] /\left(x^{2}, y^{2}, x y\right) .
$$

Corollary 7. The ring $S_{n}$ could never be a morphic ring.

Proof. We proof the corollary by showing that the element of the type $x_{1}+x_{2}$ could never be a morphic element.

Assume $x_{1}+x_{2}$ is morphic. There must be an element $\beta=a_{0}{ }^{\prime}+\sum_{i=1}^{n} a_{i}{ }^{\prime} x_{i}$ such that

$$
l\left(x_{1}+x_{2}\right)=R x_{1}+R x_{2}+\cdots+R x_{n}=S_{n} \beta
$$




$$
=\left\{\left(a_{0} a_{0}{ }^{\prime}+\sum_{i=1}^{n}\left(a_{0} a_{i}{ }^{\prime}+a_{i} a_{0}{ }^{\prime}\right) x_{i} \mid a_{0}, a_{i},, a_{0}{ }^{\prime}, a_{i}{ }^{\prime} \in R\right\} .\right.
$$

Then $a_{0}{ }^{\prime}=0, a_{i}{ }^{\prime} \in U(R)$, and $l(\beta)=R x_{1}+R x_{2}+\cdots+R x_{n}$. But we have

$$
\begin{aligned}
S_{n}\left(x_{1}+x_{2}\right) & =\left\{\left(a_{0}+\sum_{i=1}^{n} a_{i} x_{i}\right)\left(x_{1}+x_{2}\right) \mid a_{0}, a_{i} \in R\right\} \\
& =\left\{a_{0} x_{1}+a_{0} x_{2} \mid a_{0} \in R\right\} \neq l(\beta) .
\end{aligned}
$$

This is a contradiction.

Hence the element of the type $x_{1}+x_{2}$ could never be a morphic element, and the ring $S_{n}$ could never be a morphic ring.

Further, we know that every idempotent $e_{s_{n}} \in S_{n}$ is morphic. By Theorem 6, we assume $e_{s_{n}}=u_{s_{n}} e$, then

$$
e_{s_{n}}^{2}=u_{s_{n}} e u_{s_{n}} e=u_{s_{n}}^{2} e=u_{s_{n}} e \text {, so } u_{s_{n}} e=e \text {, that is } e_{s_{n}}=e \text {. }
$$

Corollary 8. Let $R$ be a strongly regular ring. Then the idempotents in $S_{n}$ are just the idempotents in $R$.

Proof. Here we give another normal way to prove the corollary, and take $n=2$ for example.

Suppose $\alpha=a+b x_{1}+c x_{2} \in S_{2}$ is idempotent, we get

$$
\left(a+b x_{1}+c x_{2}\right)^{2}=a^{2}+(a b+b a) x_{1}+(a c+c a) x_{2}=a+b x_{1}+c x_{2}
$$

then

$$
a^{2}=a, a b+b a=b, a c+c a=c
$$

thus

$$
a b+a b a=a b, a b a=0 .
$$

Since $R$ is strongly regular, every idempotent is in center. Then $a b=b a=$ $a b a=0$ thus $b=0$. With the same method we can get $c=0$.

Finally, we give an example that $R$ is unit regular, and an element $\alpha \in S_{n}$ is morphic but not of the form $u_{s_{n}} e$.

Example 9. Let $R=M_{2}\left(\mathbb{Z}_{2}\right)$, considering the element $\alpha=\left(\begin{array}{ll}1 & 0 \\ 0 & 0\end{array}\right)+\left(\begin{array}{ll}0 & 1 \\ 0 & 0\end{array}\right) x \in$ $S_{2}$. We show that $\alpha$ is a morphic element but could not be the form of $u_{s_{2}} e$.

Proof. First verify the morphic property of $\alpha$, denote

$$
A=\left(\begin{array}{ll}
1 & 0 \\
0 & 0
\end{array}\right), B=\left(\begin{array}{ll}
0 & 1 \\
0 & 0
\end{array}\right)
$$

and we have

$$
\begin{aligned}
A^{2} & =A, A B+B A=B \\
\Rightarrow \alpha^{2}=(A+B x)^{2} & =A^{2}+(A B+B A) x=A+B x=\alpha .
\end{aligned}
$$

Thus $\alpha$ is an idempotent in $S_{2}$, so it is a morphic element. But if

$$
\alpha=u_{s_{2}} e=(u+a x+b y) e=u e+a e x
$$




$$
\Rightarrow A=u e, B=a e,
$$

then

$$
B=a u^{-1} A=\left(\begin{array}{l}
* * \\
* *
\end{array}\right)\left(\begin{array}{ll}
1 & 0 \\
0 & 0
\end{array}\right)=\left(\begin{array}{ll}
* & 0 \\
* & 0
\end{array}\right) \neq\left(\begin{array}{ll}
0 & 1 \\
0 & 0
\end{array}\right) .
$$

This is a contradiction.

\section{References}

[1] V. Camillo, W. K. Nicholson, and Z. Wang, Left quasi-morphic rings, J. Algebra Appl. 7 (2008), no. 6, 725-733.

[2] J. Chen and Y. Zhou, Morphic rings as trivial extensions, Glasg. Math. J. 47 (2005), no. $1,139-148$.

[3] K. R. Goodearl, von Neumann regular rings, in: Monographs and Studies in Mathematics, Pitman, Boston, Mass, London, 1979.

[4] T. Y. Lam, A First Course in Noncommutative Rings, Second ed., Grad. Texts in Math., vol. 131, Springer-Verlag, New York, 2001.

[5] T.-K. Lee and Y. Zhou, Morphic rings and unit regular rings, J. Pure Appl. Algebra 210 (2007), no. 2, 501-510.

[6] W. K. Nicholson and E. Sánchez Campos, Rings with the dual of the isomorphism theorem, J. Algebra 271 (2004), no. 1, 391-406.

[7] — Morphic modules, Comm. Algebra 33 (2005), no. 8, 2629-2647.

KAI LONG

Department of Mathematics

Aviation University of Air Force

130022, Changchun, Jilin, P. R. China

E-mail address: 1kkkkkkkk@hotmail.com

QICHUAN WANG

No. 8, Anningqu Road, 830001, Urumqi, Xinjiang, P. R. China

E-mail address: wangqichuan1026@163.com

LiANGGUI FENG

COllege OF SCIENCE

National University of Defense Technology

410073, Changsha, Hunan, P. R. China

E-mail address: fenglg2002@sina.com 